\title{
SOME INVOLUTORIAL LINE TRANSFORMATIONS INTERPRETED AS POINTS OF $V_{2}$ OF $S_{5}{ }^{*}$
}

BY J. M. CLARKSON

\section{Introduction. Consider the identity}

$$
V_{2}(x) \equiv x_{1} x_{4}+x_{2} x_{5}+x_{3} x_{6} \equiv 0
$$

existing among the Plücker coordinates $x_{1}, x_{2}, \cdots, x_{6}$ of a line in $S_{3}$ as the equation of a quadratic hypersurface in $S_{5}$. The existence of a $(1,1)$ correspondence between the lines of $S_{3}$ and the points of $V_{2}$ is well known, as is also the representation of ruled surfaces in $S_{3}$ by curves of the same orders and genera on $V_{2} \cdot \dagger$

To a bundle of lines in $S_{3}$ corresponds a plane of points on $V_{2}$, and to a plane field of lines corresponds a plane of points on $V_{2}$, called $\omega$-planes and $\rho$-planes, respectively, throughout this paper. The two systems of planes are each $\infty^{3}$, and there are certain relations among them. Two planes of either system have always one and only one point in common, corresponding to the line common to the two representative bundles or plane fields; and a plane of the $\omega$-system meets a plane of the $\rho$-system either in no point or in a line of points, corresponding to the flat pencil common to a bundle and a plane field of $S_{3}$ when the vertex of the bundle lies on the plane of the field.

Line transformations of $S_{3}$ are, therefore, point transformations on $V_{2}$. When the point transformations on $V_{2}$ are nonlinear, their fundamental elements may be of dimension $0,1,2$, 3 , and their images, or the principal elements in the transformations, may be of dimension $1,2,3,4$. The transformations considered below are birational, but since the equation of $V_{2}$ does not enter in to the discussion of their birationality, we conclude that they are Cremona transformations for all of $S_{5}$.

2. Three Involutorial Transformations on $V_{2}$.

Case 1. J. DeVriesł discusses synthetically several involutions

* Presented to the Society, March 26, 1932.

$\dagger$ See, for example, W. L. Edge, Ruled Surfaces.

$\ddagger$ Proceedings, Koninklijke Akademie van Wetenschappen te Amsterdam, vol. 22 (1920), pp. 478-481, 634-640. 
among the rays of space. It shall be our purpose here to obtain analytic formulations of these transformations on $V_{2}$ in $S_{5}$. To this end, let us consider the four flat pencils $\left(A_{i}, \alpha_{i}\right)$ in the planes $\alpha_{i}$ and with vertices at $A_{i}$. Consider the planes $\alpha_{i}$ as being the faces of the tetrahedron of reference so that $\alpha_{i} \equiv z_{i}=0$, where $z_{i}$ are the variable coordinates of a point of $S_{3}$, and choose for the points $A_{1}, A_{2}, A_{3}, A_{4}$ the points $(0,1,1,1),(1,0,1,1)$, $(1,1,0,1),(1,1,1,0)$ respectively. The pencils $\left(A_{i}, \alpha_{i}\right)$ are therefore independent and the Plücker coordinates of a line of each are

$$
\left\{\begin{array}{l}
a_{1} \equiv\left[\left(\lambda_{1}+\lambda_{2}\right),-\lambda_{1},-\lambda_{2}, 0,0,0\right] \\
a_{2} \equiv\left[-\left(\mu_{1}+\mu_{2}\right), 0,0,0, \mu_{2},-\mu_{1}\right] \\
a_{3} \equiv\left[0,-\left(l_{1}+l_{2}\right), 0,-l_{2}, 0, l_{1}\right], \\
a_{4} \equiv\left[0,0,-\left(m_{1}+m_{2}\right), m_{2},-m_{1}, 0\right]
\end{array}\right.
$$

where the $\lambda_{j}, \mu_{j}, l_{j}, m_{j}$, represent parameters and the $a_{i}$ lines from the pencils $\left(A_{i}, \alpha_{i}\right)$ respectively.

An arbitrary line $(y)$ will determine a definite line of each pencil, and these four lines, in general mutually skew, will have another transversal line $(x)$. Conversely, the line $(x)$ determines the line $(y)$, and hence if we consider the variables $\left(x_{i}\right),\left(y_{i}\right)$ as coordinates of points in $S_{5}$, we have an involution of $S_{5}$ which leaves $V_{2}$ invariant. Such a line $(y)$ determines in the given four pencils the lines

$$
\left\{\begin{array}{l}
a_{1} \equiv\left[\left(y_{5}-y_{6}\right),\left(y_{6}-y_{4}\right),\left(y_{4}-y_{5}\right), 0,0,0\right], \\
a_{2} \equiv\left[-\left(y_{2}+y_{3}\right), 0,0,0,\left(y_{3}+y_{4}\right),\left(y_{4}-y_{2}\right)\right], \\
a_{3} \equiv\left[0,\left(y_{1}+y_{3}\right), 0,\left(y_{3}-y_{5}\right), 0,\left(y_{1}+y_{5}\right)\right], \\
a_{4} \equiv\left[0,0,-\left(y_{1}+y_{2}\right),\left(y_{2}+y_{6}\right),\left(y_{6}-y_{1}\right), 0\right] .
\end{array}\right.
$$

Recalling that the lines meeting a given line $a$ of $S_{3}$ are represented by the intersection with $V_{2}$ of the $S_{4}$ tangent to it at the point which represents $a$, we have the equations

$$
\left\{\begin{array}{l}
\left(y_{5}-y_{6}\right) x_{4}+\left(y_{6}-y_{4}\right) x_{5}+\left(y_{4}-y_{5}\right) x_{6}=0 \\
\left(y_{3}+y_{4}\right) x_{2}+\left(y_{4}-y_{2}\right) x_{3}-\left(y_{2}+y_{3}\right) x_{4}=0 \\
\left(y_{3}-y_{5}\right) x_{1}+\left(y_{1}+y_{5}\right) x_{3}+\left(y_{1}+y_{3}\right) x_{5}=0 \\
\left(y_{2}+y_{6}\right) x_{1}+\left(y_{6}-y_{1}\right) x_{2}-\left(y_{1}+y_{2}\right) x_{6}=0
\end{array}\right.
$$

of four $S_{4}$ 's tangent to $\overrightarrow{V_{2}}$ at the points $a_{1}, a_{2}, a_{3}, a_{4}$ respectively. 
The problem of solving four linear and one quadratic equations in six unknowns is the same as that discussed by $\mathrm{V}$. Snyder,* in which he determines the two lines common to four linear line-complexes. Using his method of solution we find

$$
\begin{array}{lll}
\rho x_{1}=w_{1} Q \cdot R, & \rho x_{2}=w_{2} S \cdot R, & \rho x_{3}=w_{3} S \cdot Q, \\
\rho x_{4}=w_{4} S \cdot U, & \rho x_{5}=w_{5} U \cdot Q, & \rho x_{6}=w_{6} U \cdot R,
\end{array}
$$

where the $w_{i}$ are linear and $Q, R, S, U$ are cubic in $y_{j}$. Thus the transformation (4) is an involution of order seven.

Since $V_{2}$ is invariant under (4), $x_{i}$ must satisfy $V_{2}(x)=0$. But if this be true, $V_{2}(w)=0$ also.

If $Q(y)=0$, then in (4) $x_{1}=x_{3}=x_{5}=0$. Hence any point in the plane $x_{1}=x_{3}=x_{5}=0$ on $V_{2}$ is transformed into the intersection of the cubic hypersurface $Q(x)=0$ and $V_{2}(x)=0$. Likewise any point in the plane $x_{1}=x_{2}=x_{6}=0$ is transformed into the intersection of $R(x)=0$ and $V_{2}(x)=0$, any point in the plane $x_{2}=x_{3}=x_{4}=0$ is transformed into the intersection of $S(x)=0$ and $V_{2}(x)=0$, and any point in the plane $x_{4}=x_{5}=x_{6}=0$ is transformed into the intersection of $U(x)=0$ and $V_{2}(x)=0$. These planes on $V_{2}$ are therefore fundamental elements of (4). They are $\rho$-planes, being the representation on $V_{2}$ of the lines of the planes $\alpha_{1}, \alpha_{2}, \alpha_{3}, \alpha_{4}$ respectively. Dually, the $\omega$-planes, representative of the bundles of lines of $S_{3}$ with vertices at $A_{1}, A_{2}, A_{3}, A_{4}$ respectively, are fundamental elements of (4).

There are $\rho$-planes and $\omega$-planes which carry singular flat pencils. Consider the $\rho$-plane which represents the lines of the plane $A_{1} A_{2} A_{3}$ in $S_{3}$. Its equations are

$$
x_{4}+x_{5}-2 x_{6}=0 ; 2 x_{2}-x_{3}+x_{4}=0 ; 2 x_{1}+x_{3}+x_{5}=0 ;
$$

and the point $(0,0,1,1,-1,0)$ which lies in this plane is such that every line in the pencil with this point as vertex and $\left(4 \mathrm{a}_{1}\right)$ as base is singular. In $S_{3}$, plane $A_{1} A_{2} A_{3}$ meets $\alpha_{4}$ in a line. Every line $t$ in $A_{1} A_{2} A_{3}$ meets the intersection $A_{1} A_{2} A_{3}, \alpha_{4}$ in a point and so determines a line $a_{4}$. The line $t$ meets each of the intersections $A_{1} A_{2} A_{3}, \alpha_{1} ; A_{1} A_{2} A_{3}, \alpha_{2} ; A_{1} A_{2} A_{3}, \alpha_{3}$. These intersections are lines of the pencils $\left(A_{i}, \alpha_{i}\right),(i=1,2,3)$ respectively, and $t^{\prime}$, the conjugate of $t$ in (4), is any one of the lines of the pencil in the plane

* This Bulletin, vol. 3 (1897), pp. 247-250. 
$A_{1} A_{2} A_{3}$ with vertex $t, a_{4}$. Hence, the pencil is singular. But, on $V_{2}$ in $S_{5}$, a pencil of lines of $S_{3}$ is represented by a line and since all of the pencils $\left(t, a_{4} ; A_{1} A_{2} A_{3}\right)$ have in common the line $A_{1} A_{2} A_{3}, \alpha_{4}$ whose representation on $V_{2}$ is the point $(0,0,1,1,-1,0)$, the lines on $V_{2}$ which represent the pencils $\left(t, a_{4} ; A_{1} A_{2} A_{3}\right)$ intersect in this point. In like manner, each of the planes

$\left(4 \mathrm{a}_{2}\right) x_{4}-2 x_{5}+x_{6}=0 ; 2 x_{1}+x_{2}-x_{6}=0 ; x_{2}+2 x_{3}+x_{4}=0$; $\left(4 \mathrm{a}_{3}\right) 2 x_{4}-x_{5}-x_{6}=0 ; x_{1}+2 x_{3}-x_{5}=0 ; x_{1}+2 x_{2}+x_{6}=0$; $\left(4 \mathrm{a}_{4}\right) x_{2}-x_{3}-2 x_{4}=0 ; x_{1}-x_{3}+2 x_{5}=0 ; x_{1}-x_{2}-2 x_{6}=0$; carries a singular pencil of lines with vertices at the points $(0,1,0,-1,0,1),(1,0,0,0,1,-1),(1,1,1,0,0,0)$ respectively. Dually, each of the $\omega$-planes on $V_{2}$ which represent the bundles of lines of $S_{3}$ with vertices at $\alpha_{1} \alpha_{2} \alpha_{3}, \alpha_{1} \alpha_{2} \alpha_{4}, \alpha_{1} \alpha_{3} \alpha_{4}, \alpha_{2} \alpha_{3} \alpha_{4}$ carries a singular pencil of lines.

The involution (4) has fundamental points which are transformed into quadric surfaces lying on $V_{2}$. Consider the point $(1,0,0,0,0,0)$. If $U=S=0$, then application of (4) gives this point. But, if

$$
x_{3}-x_{5}=0 ; x_{2}+x_{6}=0,
$$

then

$$
U(x)=S(x)=0 .
$$

But $\left(5 \mathrm{a}_{1}\right)$ are the equations of a three-space common to the four-spaces $x_{1}=0, x_{4}=0$, both of which are tangent to $V_{2}$. Thus $\left(5 \mathrm{a}_{1}\right)$ are the equations of a quadric surface on $V_{2}$. In like manner we find that the points

$(0,1,0,0,0,0),(0,0,1,0,0,0),(0,0,0,1,0,0),(0,0,0,0,1,0),(0,0,0,0,0,1)$

are fundamental points with quadric surfaces as images. Dually, the points

$$
\begin{aligned}
& (0,1,-1,1,1,1),(-1,0,1,1,1,1),(1,-1,0,1,1,1) \\
& (1,1,-1,1,-1,0),(1,-1,1,-1,0,1),(-1,1,1,0,1,-1)
\end{aligned}
$$

are fundamental with quadrics as images.

The locus of invariant points of (4) is the intersection of $V_{2}$ and a hypersurface of order four. Snyder* showed that the two lines common to four linear complexes are coincident if, and only if, the combinant

\footnotetext{
* Loc. cit.
} 


$$
\Delta \equiv\left|\begin{array}{cccc}
A_{11} & A_{12} & A_{13} & A_{14} \\
A_{21} & A_{22} & A_{23} & A_{24} \\
A_{31} & A_{32} & A_{33} & A_{34} \\
A_{41} & A_{42} & A_{43} & A_{44}
\end{array}\right|
$$

of the system of complexes having these four as base vanishes. Equations (3) are the equations of four special linear complexes, and the combinant of the system with (3) as base is $\Delta$, where

$$
A_{i k} \equiv a_{i 1} a_{k 4}+a_{i 2} a_{k 5}+a_{i 3} a_{k 6}+a_{i 4} a_{k 1}+a_{i 5} a_{k 2}+a_{i 6} a_{k 3},
$$

where now $a_{i j}$ represents the coefficient of $x_{j}$ in the $i$ th equation of (3).

After making the substitution indicated above and using the fact that $V_{2}(w)=0$, we obtain

$$
\begin{aligned}
\Delta(x) & \equiv\left[w_{2}(x) w_{5}(x) x_{3} x_{6}-w_{3}(x) w_{6}(x) x_{2} x_{5}\right]^{2} \\
& \equiv\left[w_{1}(x) w_{4}(x) x_{3} x_{6}-w_{3}(x) w_{6}(x) x_{1} x_{4}\right]^{2} \\
& \equiv\left[w_{1}(x) w_{4}(x) x_{2} x_{5}-w_{2}(x) w_{5}(x) x_{1} x_{4}\right]^{2} .
\end{aligned}
$$

Thus, the locus of invariant points of (4) is

$$
w_{1} w_{4} x_{2} x_{5}-w_{2} w_{5} x_{1} x_{4}=0 ; V_{2}(x)=0 .
$$

Among the lines of $S_{3}$, (4) transforms a pencil $(T, \tau)$ into a ruled surface $R_{7}$ of order seven. Four lines of the pencil $(T, \tau)$ are generators of $R_{7}$, for (8) is the equation of a line-complex of order four, of which any pencil carries four lines. On $R_{7}$ there is a double curve of order 15 and genus 6 . $^{*}$ The plane $\tau$ intersects $R_{7}$ in four lines $t_{1}, t_{2}, t_{3}, t_{4}$ of $(T, \tau)$ and a curve of order 3 which must pass through $T$ since every generator of $R_{7}$ meets five others. Hence there are two generators of $R_{7}$ not in $\tau$ met by each of the lines $t_{i}$. This cubic curve is rational, since the surface $R_{7}$ is rational, and has a double point which is not on any of the lines $t_{i}$. The double curve of order 15 meets $\tau$ in 15 points, of which 8 are the pairs of points in which $t_{i}$ meet the residual cubic $\left[R_{7}, \tau\right]$. The six pairs of generators of $R_{7}$ among $t_{i}$ show that the double curve has a 6 -fold point at $T$. Project this curve from $T$ upon a plane. We obtain thus a plane curve $\rho_{9}$ of

\footnotetext{
* See W. L. Edge, Ruled Surfaces, pp. 9 and 29.
} 
order 9 and genus 6 . The line of intersection of $\tau$ and the plane of section passes through four double points of $\rho_{9}$ and hence meets $\rho_{9}$ in one other point, the point where the line of $\tau$ through $T$ and the double point of $\left[R_{7}, \tau\right]$ meets the plane of section. Now, $R_{7}$ has 10 triple points, ${ }^{*}$ of which the four-fold point $T$ counts for four. Hence there are six others which are also three-fold on the double curve. These six triple points project in to triple points of $\rho_{9}$, and since $\rho_{9}$ is of genus 6 and has four double points (mentioned above), these six triple points are just sufficient to complete the singularities of $\rho_{9}$.

On $V_{2}$ in $S_{5}$ there are six $\omega$-planes with three intersections each with $C_{7}$ (representation of $R_{7}$ ). These are the $\omega$-planes which represent the bundles of lines through the triple points of $R_{7}$. Likewise, there are six $\rho$-planes trisecant to $C_{7}$, representing the lines of the tritangent planes of $R_{7}$. There is one plane of each system having four intersections with $C_{7}$, representing the bundle $T$ and the plane field $\tau$ respectively. There are only $\infty^{1}$ planes of $V_{2}$ which meet $C_{7}$ twice, and $\infty^{2}$ which meet $C_{7}$ once. They represent the lines of the bundles and of the bitangent planes at points of the double curve, and the lines of the bundles and of the tangent planes at the ordinary points of $R_{7}$ respectively.

A bundle of lines $T$ is transformed into a line-congruence of order seven formed by the generators of the ruled surfaces $R_{7}$ belonging to the singly infinite system arising from the pencils of lines through $T$. The invariant lines of the transformation make up a quartic cone $K_{4}$ which must pass through the points $A_{1}, A_{2}, A_{3}, A_{4} ; \alpha_{1}, \alpha_{2}, \alpha_{3}, \alpha_{1}, \alpha_{2}, \alpha_{4} ; \alpha_{1}, \alpha_{3}, \alpha_{4}, \alpha_{2}, \alpha_{3}, \alpha_{4}$, since each line $t$ of the bundles having these points as vertices is transformed into either a quadric regulus to which $t$ belongs or into a pencil of lines containing $t$.

On $V_{2}$ in $S_{5}$ the $\omega$-plane which represents the bundle $T$ is transformed into a surface of order seven which meets the $\omega$ plane in a curve of order four, the representation on $V_{2}$ of $K_{4}$ of $S_{3}$.

Again, a linear complex of lines in $S_{3}$ is transformed into a line-complex of order seven, and the two complexes have in common a congruence of order four. On $V_{2}$ in $S_{5}$ the intersection of

* The number of triple points of a ruled surface of order $n$, genus, $p$, is $(n-4)[(n-2)(n-3) / 6-p]$. See Edge, loc. cit., p. 31 . 
$V_{2}$ with a general hyperplane is transformed into the intersection of $V_{2}$ with a hypersurface of order seven. The invariant points form a surface of order four.

CASE 2. Let us now consider the involution defined among the lines of $S_{3}$ (and hence among the points of $V_{2}$ in $S_{5}$ ) by the transversals of one regulus of a quadric surface and the lines of two flat pencils whose bases and whose vertices are not conjugate as to the quadric. The bases are not tangent to, nor do the vertices lie on the quadric.

Choose as the quadric surface

$$
z_{1}^{2}-z_{2}^{2}+z_{3}^{2}-z_{4}^{2}=0,
$$

and on this the regulus

$$
\frac{z_{1}+z_{2}}{z_{3}+z_{4}}=-\frac{z_{3}-z_{4}}{z_{1}-z_{2}}=k,
$$

and as the bases of the pencils choose the planes

$$
z_{1}-2 z_{2}=0 ; z_{2}-2 z_{3}=0,
$$

and as their vertices the points $(2,1,0,0) ;(0,2,1,0)$ respectively.

The involution defined by this system is

$$
x_{i}=\phi_{i}(y), \quad(i=1, \cdots, 6),
$$

where the $\phi_{i}$ are of degree five in $y_{j}$. The involution is rational.

In $S_{5}$, (9) defines a point transformation, involutorial and of order five. The $\rho$-planes which represent the plane fields $\alpha_{i}$ of $S_{3}$ and the $\omega$-planes representing the bundles $A_{i}$ are transformed into the intersection of $V_{2}$ with certain quadric hypersurfaces, and the points representative of the lines $\alpha_{1} \alpha_{2}, A_{1} A_{2}$ of $S_{3}$ are transformed into ordinary quadric surfaces on $V_{2}$. Also, the conic which represents the second regulus of the quadric surface with which we started in $S_{3}$ is transformed into the intersection of $V_{2}$ with a quartic hypersurface.

By the same method employed in Case 1, the locus of invariant points is found to be of order three and to contain the conic representative of the second regulus.

A general line on $V_{2}$ is transformed into a rational curve of order five meeting the line in three points. A general plane of either system is transformed into a surface of order five on $V_{2}$ 
meeting the plane in a cubic curve, and a general linear threedimensional locus on $V_{2}$ is transformed into a three-dimensional locus of order five meeting the linear locus in a cubic surface.

Case 3. The involution defined by the transversals of two generators from one regulus of each of two quadric surfaces which have no generator in common is of order three with an invariant complex of order two. Among the invariant lines are the generators of the other regulus on each quadric.

The transformation on $V_{2}$ has for fundamental elements the two conics representative of the second reguli of the quadrics in $S_{3}$ and a ruled surface $R_{4}$ of order four, each of whose points is transformed into the entire generator of $R_{4}$ on which it lies. The surface $R_{4}$ is the representation in $S_{5}$ of the $(4,4)$ congruence of lines of $S_{3}$ formed by $\infty^{1}$ pencils whose vertices are on the curve of intersection of the two quadrics and whose bases are the planes determined by the intersecting generators of the reguli considered on the two quadrics.

A general line on $V_{2}$ is transformed into a rational curve of order three meeting the line in two points. A general plane of either system is transformed into a cubic surface which meets the plane in a conic, and a general linear three-dimensional locus is transformed into a three-dimensional locus of order three meeting the linear locus in a quadric surface.

Other properties of this transformation have also been obtained by A. R. Williams* by a partly different method; they will not be repeated here.

Cornell University

* Williams, The transformation of lines of space by means of two quadratic reguli, the present issue of this Bulletin, vol. 38 (1932), p. 554. 